\title{
Prevalence of antibiotic prescription in southern Italian outpatients: real-world data analysis of socioeconomic and sociodemographic variables
} at a municipality level

This article was published in the following Dove Press journal: ClinicoEconomics and Outcomes Research

\author{
Veronica Russol,* \\ Valeria Marina Monetti ${ }^{1, *}$ \\ Francesca Guerriero' \\ Ugo Trama \\ Antonella Guida ${ }^{3}$ \\ Enrica Menditto' \\ Valentina Orlando' \\ 'CIRFF, Center of \\ Pharmacoeconomics, University \\ of Naples Federico II, ${ }^{2}$ Regional \\ Pharmaceutical Unit, ${ }^{3}$ Directorate- \\ General for Protection of Health, \\ Campania Region, Naples, Italy
}

*These authors contributed equally to this work

Correspondence: Enrica Menditto CIRFF, Center of Pharmacoeconomics, University of Naples Federico II, Via Domenico Montesano 49, 80I3I Naples, Italy

Tel +3981678660

Fax +3981678658

Email enrica.menditto@unina.it
Purpose: The aim of this study was to analyze the geographic variation in systemic antibiotic prescription at a regional level and to explore the influence of socioeconomic and sociodemographic variables.

Methods: This study was a retrospective analysis of reimbursement pharmacy records in the outpatient settings of Italy's Campania Region in 2016. Standardized antibiotic prescription rates were calculated at municipality and Local Health Unit (LHU) level. Antibiotic consumption was analyzed as defined daily doses (DDD)/1000 inhabitants per day (DID). Logistic regression was performed to evaluate the association between antibiotic prescription and sociodemographic and socioeconomic determinants at a municipality level.

Results: The average antibiotic prevalence rate was $46.8 \%$. At LHU level, the age-adjusted prevalence rates ranged from $41.1 \%$ in Benevento to $51.0 \%$ in Naples2. Significant differences were found among municipalities, from $15.2 \%$ in Omignano (Salerno LHU [Sa-LHU]) to $61.9 \%$ in Moschiano (Avellino [Av-LHU]). The geographic distribution also showed significant differences in terms of antibiotic consumption, from 6.7 DID in Omignano to 41.6 in San Marcelino (Caserta [Ce-LHU]). Logistic regression showed that both municipality type and average annual income level were the main determinants of antibiotic prescription. Urban municipalities were more than eight times as likely to have antibiotic high prevalence rates compared to rural municipalities (adjusted odds ratio [OR]: 8.62; 95\% confidence interval [CI]: 4.06-18.30, $P<0.001$ ). Low average annual income level municipalities were more than eight times as likely to have antibiotic high prevalence rates compared to high average annual income level municipalities (adjusted OR: 8.48; 95\% CI: 3.45-20.81, $P<0.001$ ).

Conclusion: We provide a snapshot of Campania's antibiotic consumption, evidencing the impact of both socioeconomic and sociodemographic factors on the prevalence of antibiotic prescription. The observed intraregional variability underlines the lack of shared therapeutic protocols and the need for careful monitoring. Our results can be useful for decision makers to plan educational interventions, thus optimizing health resources and improving rational drug use. Keywords: retrospective databases, drug utilization, appropriateness

\section{Introduction}

Antibiotic consumption in Europe has increased over the last few years, making them the most prescribed drugs in outpatient populations. ${ }^{1,2}$

Antibiotics may be prescribed for the treatment of various diseases, ${ }^{3-7}$ but more than one-third of Europeans take them unnecessarily or without a prescription, contrarily to European Union (EU) recommendations. ${ }^{8}$ 
Antibiotic overuse and misuse contribute not only to the development of resistance but also to treatment failure and increase in mortality. Different studies have shown a correlation between the irrational use of antimicrobial drugs and antibiotic resistance to bacterial pathogens. ${ }^{9-11}$

The World Health Organization (WHO) advocates the correct use of antibiotics to avoid antibiotic resistance, which has reached alarming levels worldwide. ${ }^{8}$ Thus, the interest in the epidemiology of antibiotic use has increased.

Within Europe, major differences in antibiotic consumption rates have been noted. ${ }^{12-16}$ These geographical variations have been attributed to socioeconomic (eg, financial wellbeing and access to health insurance), sociodemographic (eg, urbanization), and cultural (eg, educational level, prescribing norms, and patient demands) factors. ${ }^{17,18}$ Several studies show that the Italian consumption of systemic antibiotics is higher than the European average, both in hospitals and in the outpatient population., ${ }^{2,19,20}$ Furthermore, there is evidence that antibiotic prescription rates vary among different Italian regions and also among areas within the same region, showing that the differences in antibiotic use are influenced by both national policies and geographical typology. ${ }^{21-26}$

There is still a considerable variability between antibiotic consumption in southern (44.9\%) and northern (31.6\%) Italian regions. Particularly, antibiotic consumption in Campania is the highest in Italy. ${ }^{27}$

Italian health policies have been decentralized at a regional level since 2001. However, regional antibiotic prescribing patterns in southern Italy have not been investigated in depth. Administrative health-related databases, such as pharmaceutical records, can be useful tools to explore drug exposure in a real-world setting. ${ }^{28}$

The aim of this study is to evaluate the prevalence of systemic antibiotic use at the individual municipalities in southern Italy, considering the influence of socioeconomic and sociodemographic variables.

\section{Methods}

\section{Study design}

We conducted a descriptive cross-sectional drug use study according to the Strengthening the Reporting of Observational Studies in Epidemiology (STROBE) guidelines. ${ }^{29}$

\section{Study setting}

The Italian National Health Service (NHS) has been decentralized at national, regional, and local levels, since 2001. Campania, one of the largest Italian regions situated in the south of the country, had a population of 5,850,850 inhabitants up to January 1, 2016 (according to http://demo. istat.it/pop2016/index.html). As all other Italian regions, it provides health care services (free or at a nominal charge) to all citizens and legal foreign residents through Local Health Units (LHUs). Each LHU corresponds to a geographic area in Campania and is constituted by health care districts, which aggregate different municipalities. There are five geographic areas in Campania: Naples (including three LHUs, such as $\mathrm{Na}$, Na2, and Na3), Avellino LHU (Av-LHU), Benevento LHU (Bn-LHU), Caserta LHU (Ce-LHU), and Salerno LHU (Sa-LHU). Overall, there are 550 municipalities.

\section{Data source}

For this study, the following two administrative databases of Campania were analyzed: civil registry, containing demographic information (ie, age, gender, LHU, and municipality of residence) of all residents covered by the Regional Health System (RHS), and pharmaceutical databases, containing records of the drugs dispensed by retail pharmacies and reimbursed by the NHS, information regarding the patient's identification code, drug code, dose, formulation, number of packages, date of prescription, date of dispensation, and drug price. Drugs were classified according to the Anatomical Therapeutic Chemical (ATC) classification system. ${ }^{30}$

The above databases had been previously validated and used to produce drug-utilization information..$^{31-32,46-52}$ Data sources were matched by record linkage analysis through a unique and anonymous personal identification code. Such code was created by a database manager, uninvolved in the data analysis, preventing patient identification. Therefore, neither ethical committee approval nor informed consent forms were required.

\section{Study drugs}

Prescribed drugs, in Italy, are categorized into the following two classes: class A includes lifesaving drugs and treatments for chronic diseases that are fully reimbursed by the NHS and class $\mathrm{C}$ includes all nonreimbursable drugs. Most antibiotic drugs fall into class A.

We conducted the analysis of all reimbursable antibiotic prescriptions dispensed by retail pharmacies in Campania between January 1, 2016, and December 31, 2016. Only systemic antibiotics belonging to the J01 subgroup, according to the ATC classification system, were included. ${ }^{30}$

\section{Study population}

The entire Campania's population (ie, 5,850,850 inhabitants) was divided into ten groups by age $(0-6,7-14,15-24,25-34$, 
35-44, 45-54, 55-64, 65-74, 75-84, and $\geq 85$ years) and distributed into the 550 municipalities. In our analysis, official data on resident population in the Italian municipalities, which are available on Demo Istat website (http://demo.istat. it/pop2016/index.html), were up to date until January 1, 2016.

\section{Study outcomes}

Prevalence was used as a measure to estimate the degree of exposure to antibiotic prescription.

Antibiotic prevalence rates were calculated, at municipality and LHU levels, as the proportion of the population who received more than one prescription per 100 inhabitants in 2016.

Prevalence rates were probably influenced by the heterogeneous demographic distribution among the age groups. Hence, they were adjusted using a direct standardization method, where the standard population (also known as reference population) was the population in Campania up to January 1, 2016:

$$
\text { Directly standardized rate }=\frac{\sum_{i=1}^{m} w_{i} \cdot T_{i}}{\sum_{i=1}^{m} w_{i}} \cdot k
$$

where $T_{i}=n_{i} / n$ is the rate in stratum $i$ of the study population, $n_{i}$ is the number of cases in stratum $i$ of the study population, $n$ is the size of the study population in stratum $i, w_{i}$ is the size of stratum $i$ of the reference population, $m$ is the number of considered stratum, and $k$ is the multiplicative constant.

Antibiotic drug consumption was expressed as the number of defined daily doses (DDD)/1000 inhabitants/day (DID) ${ }^{33}$ DDD is the assumed average maintenance dose, per day, for a drug used for its main indication in adults. ${ }^{30}$ The DID was calculated as follows: active substance divided by the number of inhabitants/1000.

\section{Covariates}

The municipalities were also classified as rural or urban to evaluate if this difference was a significant variable. ${ }^{34}$

The average annual income data were defined as the total household income for each municipality and obtained from the Ministry of Economy and Finance website (http://www. mef.gov.it/).

\section{Statistical analysis}

The age-adjusted prevalence rates were categorized into quintiles and mapped by the patient's municipality of residence.
Antibiotic consumption (DID) was also mapped for the different municipalities.

Differences in prevalence rates between each LHU and the standard population were expressed as prevalence ratios (PRs).

PRs indicate whether the prevalence rate at LHU level was higher or lower than that of the standard population. Confidence intervals (CIs) were computed using standard methods (at $95 \%$ confidence level). ${ }^{35}$

Univariate and multivariate logistic regression models were conducted to evaluate 1) the association between the highest and lowest antibiotic prevalence rates (ie, highest vs lowest quintile of prevalence) and 2) some determinants such as municipality type (rural or urban), average annual income level per capita, and number of general practitioners (GPs) and average annual medication consumption per 1000 inhabitants.

All analyses were performed using the SPSS software Version 17.1 for Windows (SPSS Inc., Chicago, IL, USA), and a $P$-value of $<0.05$ was considered to be statistically significant. Maps for antibiotic prevalence rates were generated by a custom script that uses an Application Programming Interface (API) offered by MapBox (www.mapbox.com).

\section{Ethics statement}

All procedures performed in this study were in accordance with the current national law from Italian Medicines Agency. ${ }^{45}$ The article does not contain clinical studies, and all patients' data were fully anonymized. For this type of study, formal consent is not required. Permission to use anonymized data for the present study was granted by the responsible authority, Unità del Farmaco, Regione Campania.

\section{Results Prevalence rates at LHU level}

In 2016, 2,738,118 patients in Campania received at least one antibiotic prescription. The total antibiotic prevalence rate was $46.8 \%$.

Differences were observed in age-adjusted antibiotic prevalence rates, ranging from $41.1 \%$ in Benevento to $51.0 \%$ in $\mathrm{Na} 2$. PRs ranged from 0.88 (95\% CI: 0.87-0.89) in Benevento to 1.09 (95\% CI: $1.08-1.10)$ in Na2. Figure 1 shows that three of the seven LHUs had PRs significantly higher than expected (ie, Caserta, $\mathrm{Na} 2$, and $\mathrm{Na} 3$ ) for antibiotics, while in $\mathrm{Av}, \mathrm{Na} 1$, $\mathrm{Sa}$, and $\mathrm{Bn}, \mathrm{PRs}$ were lower than expected.

\section{Prevalence rates at municipalities' level}

Figure 2A shows the distribution of antibiotic prevalence rates, in quintiles by municipality, within each LHU. 


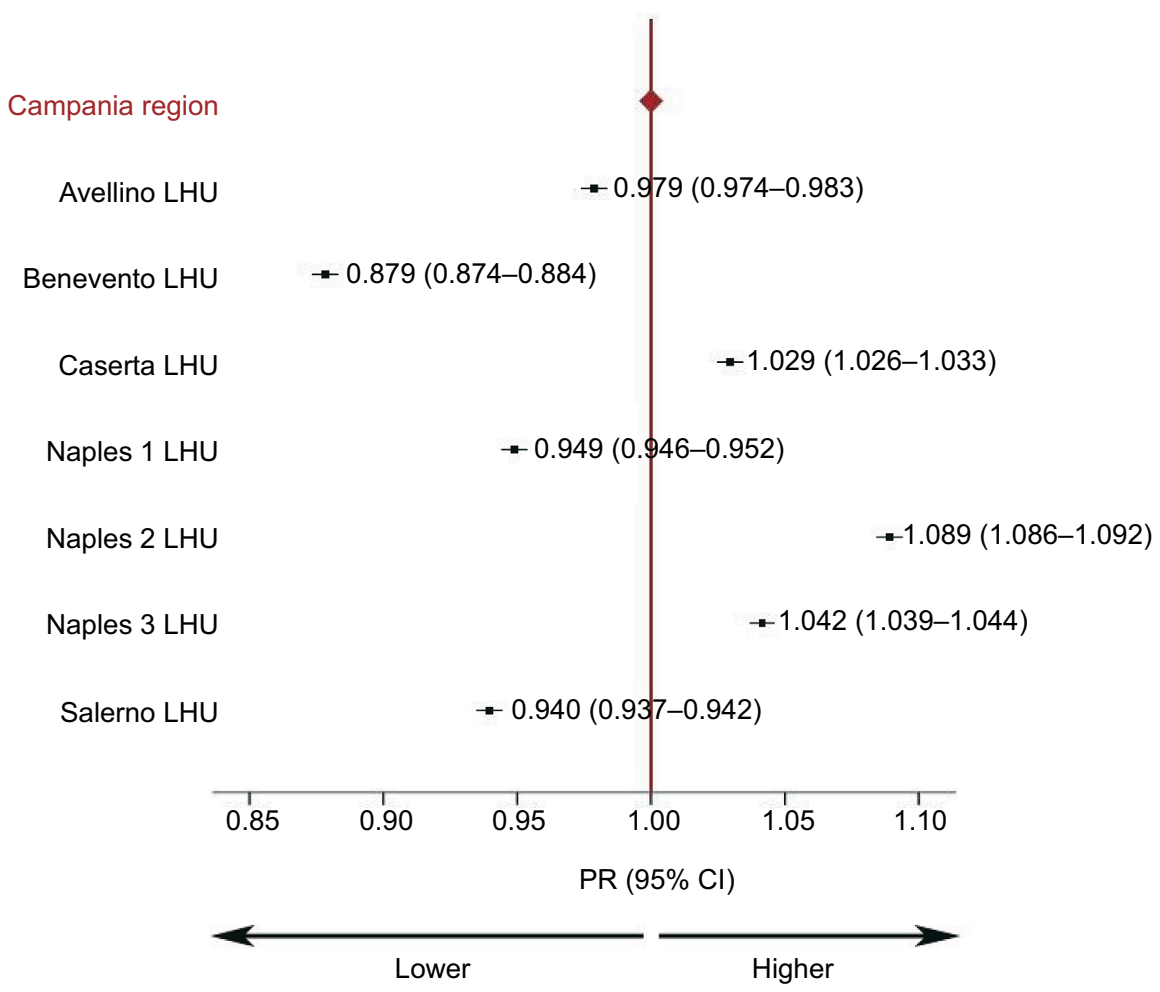

Figure I PRs at LHU level.

Note: PRs indicate differences in prevalence rates between each LHU and standard population (Campania).

Abbreviations: $\mathrm{Cl}$, confidence interval; LHU, Local Health Unit; PR, prevalence ratio.

Significant differences were found in the distribution of standardized prevalence rates between the different municipalities: from a minimum of $15.2 \%$ in Omignano (Sa-LHU) to a maximum of $61.9 \%$ in Moschiano (Av-LHU). In most municipalities of the northwestern and southern areas of Campania (ie, Benevento and Salerno areas), the prevalence rates of antibiotics were lower compared to other areas. Conversely, coastal areas around Naples and eastern Avellino showed higher prevalence rates, from 50.9 to $61.9 \%$.

Figure 2B shows the geographic distribution (by municipality) of antibiotic consumption, expressed in DID. Major differences were found between municipalities: from 6.7 DID in Omignano (Sa-LHU) to 41.6 in San Marcelino (Ce-LHU).

\section{Multivariate analysis}

Table 1 reports the results of the univariate and multivariate logistic regression analyses, which showed that two independent variables made a statistically significant contribution to the model: both municipality type and average annual income level were the main determinants of antibiotic prevalence rates. A strong significant association with prevalence rates (quintile 5 [highest] vs quintile 1 [lowest]) was municipality type. Urban municipalities (adjusted odds ratio [OR]: 8.62; 95\% CI: 4.06-18.30, $P<0.001)$ were more than eight times as likely to have antibiotic prevalence rates in quintile 5 compared to rural municipalities. Equivalent results were found for the average annual income level: municipalities with low (adjusted OR: 8.48; 95\% CI: 3.45-20.81, $P<0.001$ ) and medium (adjusted OR: 4.64; 95\% CI: 1.98-10.88, $P<0.001$ ) average annual income levels were more than eight and four times, respectively, as likely to have antibiotic prevalence rates in quintile 5 compared to high average annual income level municipalities.

\section{Discussion}

This study analyzed the prevalence of antibiotic use and consumption, at a municipality level, within Italy's Campania region. Previous studies have already evaluated intraregional variations in antibiotic prescribing patterns in Italy, ${ }^{21,23,36}$ but this study shows the relationship between antibiotic prevalence rates and socioeconomic (eg, financial and wellbeing) and sociodemographic (eg, urbanization) factors. To our knowledge, there are a limited number of similar multivariate analyses in the literature, especially at intraregional level. ${ }^{16,18,36,37}$

Differences in interregional antibiotic prescribing rates have been already described in the literature, with a higher consumption in southern Italy compared to the northern 
A

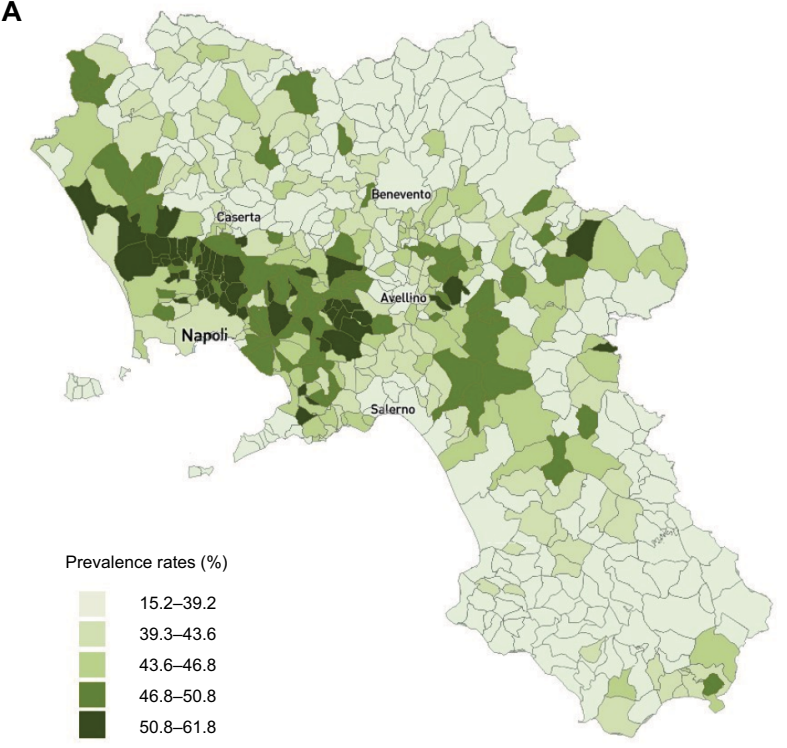

B

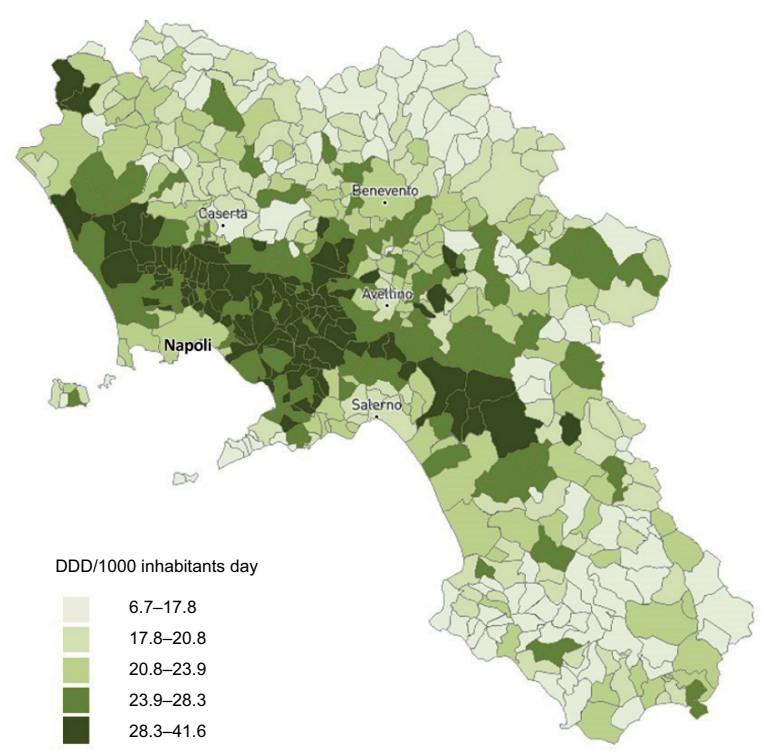

Figure 2 (A) Standardized antibiotic prevalence rates (\%). (B) Antibiotic consumption (DDD/1000 inhabitants/day). Abbreviation: DDD, defined daily doses.

Table I Multivariate analysis of antibiotic prevalence quintile 5 (highest) vs quintile I (lowest)

\begin{tabular}{|c|c|c|c|c|}
\hline Characteristics & Unadjusted OR (95\% Cl) & $P$-value & Adjusted OR (95\% Cl) & $P$-value \\
\hline \multicolumn{5}{|l|}{ Municipality type } \\
\hline Rural & Reference & & Reference & \\
\hline Urban & 7.111 (3.782-13.37I) & $<0.00 I^{*}$ & $8.62 I(4.06 I-18.30 I)$ & $<0.00 I^{*}$ \\
\hline \multicolumn{5}{|c|}{ Average annual income levels } \\
\hline High & Reference & & Reference & \\
\hline Medium & 2.734 (1.278-5.849) & $0.010^{*}$ & $4.645(1.983-10.884)$ & $<0.00 I^{*}$ \\
\hline Low & $7.862(3.423-18.058)$ & $<0.001 *$ & 8.479 (3.453-20.818) & $<0.001 *$ \\
\hline
\end{tabular}

Notes: Univariate and multivariate logistic regression models including antibiotic prevalence levels (highest vs lowest quintile of prevalence) as dependent variable and municipality type and average annual income levels as independent variables were performed. $* P<0.05$ was considered to be statistically significant.

Abbreviations: $\mathrm{Cl}$, confidence interval; OR, odds ratio.

regions. ${ }^{25,36}$ Particularly, antibiotic consumption in Campania is described as the highest in Italy. ${ }^{38} \mathrm{~A}$ similar north-south gradient has been observed at the European level. In 2016, EU population-weighted mean consumption of antibiotics for systemic use in the community was $21.9 \mathrm{DID}$, ranging from 10.4 in the Netherlands to 36.3 in Greece. Data collected by the European Surveillance of Antimicrobial Consumption (ESAC) revealed that Italy was among EU countries with higher antibiotics consumption (27 DID). ${ }^{39}$

Our study showed differences for the age-adjusted prevalence rates between the different LHUs. Large differences were found in the distribution of standardized antibiotic prevalence rates among the different municipalities (from $15.2 \%$ in Omignano, Sa-LHU, to $61.9 \%$ in Moschiano, AvLHU) and in the antibiotics consumption, expressed in DID (from 6.7 in Omignano, Sa-LHU, to 41.6 in San Marcellino, Ce-LHU). It is noteworthy that in most northwestern and southern municipalities of Campania (eg, Benevento and Salerno), prevalence rates and antibiotic consumption were lower than in coastal areas around Naples and eastern Avellino. This fact underlines that, even in settings characterized by high prevalence rates (such as Campania), there are areas with lower rates than expected and that variability is often very high, even within the same LHU. Hence, antibiotic usage is influenced by both national policies and geographical typology, as described previously. ${ }^{21-26,36}$

Our study shows that municipality type influenced antibiotic prescription prevalence. Urban municipalities were more than eight times as likely to have antibiotic prevalence rates in quintile 5 (high prevalence rates) compared to rural municipalities. The high antibiotic consumption observed in the more urbanized municipalities was probably due to a greater access to medical care, such as a higher availability of health care providers. Klein et $\mathrm{al}^{17}$ described that a higher 
number of health providers translated in an increase in antibiotic prescribing rates per capita.

Our study showed that another factor influencing the prescription rates of antibiotics was per capita income at a municipality level. Municipalities with low average annual income levels were more than eight times as likely to have antibiotic prevalence rates in quintile 5 compared to high average annual income level municipalities. These results could be of great interest for designing interventions to improve prescription patterns.

Similar findings, regarding the relationship between the prevalence of antibiotic use (in children) and annual average income, have already been observed in three Italian regions (Lombardy, Lazio, and Puglia), where children/ adolescents living in districts in the lowest quintile of annual average income were more exposed to receive an antibiotic prescription. ${ }^{36}$

Similar evidences have been described also in other EU countries, such as Germany and Switzerland. ${ }^{13,15}$ As underlined by Piovani et al, ${ }^{36}$ in countries where antibiotics prescription is reimbursed (including Italy), the confounding role of out-of-pocket drug consumption cannot be excluded, especially in studies based on administrative pharmacy records.

Sianes $\mathrm{i}^{40}$ suggested that income deprivation is a combination of other linked deprivations, including education, which is relevant and affects the appropriateness of drug use. ${ }^{16}$

The intraregional variability observed in our study can also be explained by different prescribing patterns among physicians and different local health policies. Several studies showed that the physicians' attitudes and knowledge determine the quality of antibiotic prescription. ${ }^{41}$

As already stated, geographical variations in antibiotic prescribing rates have also been observed in other EU countries. ${ }^{13-15}$ In this regard, several investigations have confirmed that socioeconomic and sociodemographic factors (eg, population, annual income, demographic structure, and cultural values) are significant determinants to explain differences in antibiotic consumption. ${ }^{17,18,42}$ Gaygisiz et al ${ }^{42}$ showed that the high variability in antibiotic use was influenced by cultural values $(65 \%)$, followed by socioeconomic $(63 \%)$ and personality (55\%) factors.

There are some limitations to our analysis. In this crosssectional study, we analyzed pharmacy records, which, although being a powerful tool, might lead to some underestimations: Pharmaceutical records do not provide information about private practice prescriptions and out-of-pocket expenditure. Therefore, the consumption of antibiotics could have been underestimated. Furthermore, we were unable to explore the prescriptions' appropriateness because the diagnosis details were unavailable from our data sources even if this is a common limitation of drug utilization studies carried out by administrative databases. Our results could be highly useful in planning policy interventions. However, it is important to be aware of the limitations of cross-sectional studies in its usefulness in making sweeping policy recommendations.

The main strengths of our study lie in providing an overview of the use and consumption of antibiotics in Campania and exploring the relationship between socioeconomic and sociodemographic factors and antibiotic consumption in a real-life setting. The analysis is useful for exploring the dynamics that are currently characterizing the use of antibiotic therapy in a regional context. Antibiotic overuse and misuse contribute to the development of resistance, treatment failure, and high health costs. Local policies, following WHO's recommendations, should provide training and information to citizens and health care professionals to optimize health resources also implementing successful elements from other EU countries' activities. ${ }^{43}$ Synergies between different actors involved in health care delivery can help in achieving better results. ${ }^{44}$ Further studies are needed to explore attitudes toward medications, which are crucial factors that could influence antibiotic use patterns.

\section{Conclusion}

Our study provides a snapshot of Campania's antibiotic drug consumption in 2016, evidencing the impact of both socioeconomic and sociodemographic factors on the prevalence of antibiotic prescription in the study's population.

Major differences were found among the different municipalities in Campania, regarding the distribution of age-standardized antibiotic prevalence rates and antibiotic consumption. Municipality type and average annual income level were the main determinants of antibiotic prescription prevalence. Our analysis underlines the lack of shared therapeutic protocols and can represent a foundational work to create them. Such protocols represent a key factor for decision-makers to improve the quality of care. Once protocols are established, they can be effectively enforced by issuing educational interventions aimed at the optimization of health resources and correct utilization of drugs.

\section{Acknowledgments}

This study is part of a set of initiatives to promote the appropriate drug use in Campania Region. EM and VO equally lead this work. 


\section{Disclosure}

The authors report no conflicts of interest in this work.

\section{References}

1. Bruyndonckx R, Hens N, Aerts M, Goossens H, Molenberghs G, Coenen S. Measuring trends of outpatient antibiotic use in Europe jointly modelling longitudinal data in defined daily doses and packages. J Antimicrob Chemother. 2014;69(7):1981-1986.

2. Adriaenssens N, Coenen S, Versporten A, et al; ESAC Project Group. European Surveillance of Antimicrobial Consumption (ESAC): outpatient antibiotic use in Europe (1997-2009). J Antimicrob Chemother. 2011;66(suppl 6):vi3-vi12.

3. Feldman C. Pneumonia in the elderly. Med Clin North Am. 2001;85(6):1441-1459.

4. Laube S. Skin infections and ageing. Ageing Res Rev. 2004;3(1):69-89.

5. Richards CL. Urinary tract infections in the frail elderly: issues for diagnosis, treatment and prevention. Int Urol Nephrol. 2004;36(3):457-463.

6. File TM Jr, Hadley JA. Rational use of antibiotics to treat respiratory tract infections. Am J Manag Care. 2002;8(8):713-727.

7. Ong DS, Kuyvenhoven MM, van Dijk L, Verheij TJ. Antibiotics for respiratory, ear and urinary tract disorders and consistency among GPs. J Antimicrob Chemother. 2008;62(3):587-592.

8. World Health Organization. Global Action Plan on Antimicrobial Resistance. 2015. Available from: http://www.wpro.who.int/entity/ drug_resistance/resources/global_action_plan_eng.pdf. Accessed May, 2017. Italian [with English abstract].

9. Goossens H, Ferech M, Vander Stichele R, Elseviers M; ESAC Project Group. Outpatient antibiotic use in Europe and association with resistance: a cross-national database study. Lancet. 2005;365(9459):579-587.

10. Andersson DI, Hughes D. Antibiotic resistance and its cost: is it possible to reverse resistance? Nat Rev Microbiol. 2010;8(4):260-271.

11. Klein E, Smith DL, Laxminarayan R. Hospitalizations and deaths caused by methicillin-resistant Staphylococcus aureus, United States, 1999-2005. Emerg Infect Dis. 2007;13(12):1840-1846.

12. Nitzan O, Low M, Lavi I, Hammerman A, Klang S, Raz R. Variability in outpatient antimicrobial consumption in Israel. Infection. 2010;38(1):12-18

13. Filippini M, Masiero G, Moschetti K. Socioeconomic determinants of regional differences in outpatient antibiotic consumption: evidence from Switzerland. Health Policy. 2006;78(1):77-92.

14. Matuz M, Benko R, Doro P, et al. Regional variations in community consumption of antibiotics in Hungary, 1996-2003. Br J Clin Pharmacol. 2006;61(1):96-100.

15. Kern WV, de With K, Nink K, Steib-Bauert M, Schroder H. Regional variation in outpatient antibiotic prescribing in Germany. Infection. 2006;34(5):269-273.

16. Koller D, Hoffmann F, Maier W, Tholen K, Windt R, Glaeske G. Variation in antibiotic prescriptions: is area deprivation an explanation? Analysis of 1.2 million children in Germany. Infection. 2013;41(1):121-127.

17. Klein EY, Makowsky M, Orlando M, Hatna E, Braykov NP, Laxminarayan $R$. Influence of provider and urgent care density across different socioeconomic strata on outpatient antibiotic prescribing in the USA. J Antimicrob Chemother. 2015;70(5):1580-1587.

18. Masiero G, Filippini M, Ferech M, Goossens H. Socioeconomic determinants of outpatient antibiotic use in Europe. Int J Public Health. 2010;55(5):469-478.

19. Morgan DJ, Okeke IN, Laxminarayan R, Perencevich EN, Weisenberg S. Non-prescription antimicrobial use worldwide: a systematic review. Lancet Infect Dis. 2011;11(9):692-701.

20. Grigoryan L, Haaijer-Ruskamp FM, Burgerhof JG, et al. Selfmedication with antimicrobial drugs in Europe. Emerg Infect Dis. 2006;12(3):452-459.

21. Franchi C, Sequi M, Bonati M, et al. Differences in outpatient antibiotic prescription in Italy's Lombardy region. Infection. 2011;39(4): 299-308.
22. Piovani D, Clavenna A, Cartabia M, Bonati M; Antibiotic Collaborative Group. The regional profile of antibiotic prescriptions in Italian outpatient children. Eur J Clin Pharmacol. 2012;68(6):997-1005.

23. Clavenna A, Sequi M, Bortolotti A, Merlino L, Fortino I, Bonati M. Determinants of the drug utilization profile in the paediatric population in Italy's Lombardy region. Br J Clin Pharmacol. 2009;67(5):565-571.

24. Piovani D, Clavenna A, Bonati M; Interregional Italian Drug Utilisation Group. Drug use profile in outpatient children and adolescents in different Italian regions. BMC Pediatr. 2013;13:46.

25. Rossignoli A, Clavenna A, Bonati M. Antibiotic prescription and prevalence rate in the outpatient paediatric population: analysis of surveys published during 2000-2005. Eur J Clin Pharmacol. 2007;63(12): 1099-1106.

26. Orzella L, Chini F, Giorgi Rossi P, Borgia P. Physician and patient characteristics associated with prescriptions and costs of drugs in the Lazio region of Italy. Health Policy. 2010;95(2-3):236-244.

27. European Center for Disease Prevention and Control (ECDC) [webpage on the Internet]. Summary of the Latest Data on Antibiotic Consumption in the EU, November 2016. 2016. Available from: https://ecdc.europa. eu/en/publications-data/summary-latest-data-antibiotic-consumptioneu-2016. Accessed May, 2017. Italian [with English abstract].

28. Schneeweiss S, Avorn J. A review of uses of health care utilization databases for epidemiologic research on therapeutics. J Clin Epidemiol. 2005;58(4):323-337.

29. STROBE [homepage on the Internet]. Statement - Checklist of Items That Should Be Included in Reports of Cohort Studies. Available from: https://www.strobe-statement.org/index.php?id=strobe-home. Accessed May, 2017. Italian [with English abstract].

30. World Health Organization [webpage on the Internet]. Collaborating Centre for Drug Statistics Methodology. Available from: https://www. whocc.no/atc_ddd_index/. Accessed May, 2017. Italian [with English abstract].

31. Casula M, Catapano AL, Piccinelli R, et al. Assessment and potential determinants of compliance and persistence to antiosteoporosis therapy in Italy. Am J Manag Care. 2014;20(5):e138-e145.

32. Cammarota S, Bruzzese D, Catapano AL, et al. Lower incidence of macrovascular complications in patients on insulin glargine versus those on basal human insulins: a population-based cohort study in Italy. Nutr Metab Cardiovasc Dis. 2014;24(1):10-17.

33. European Center for Disease Prevention and Control [webpage on the Internet]. Joint Scientific Opinion on a List of Outcome Indicators as Regards Surveillance of Antimicrobial Resistance and Antimicrobial Consumption in Humans and Food-Producing Animals. Available from: https://ecdc.europa.eu/en/publications-data/ecdc-efsa-and-ema-jointscientific-opinion-list-outcome-indicators-regards. Accessed May, 2017. Italian [with English abstract].

34. Giovanni Anania AT. Ruralità, urbanità e ricchezza dei comuni italiani. QA Riv Assoc Rossi Doria. 2008;1:71-103.

35. Newman SC. Biostatistical Methods in Epidemiology. New York: John Wiley \& Sons; 2001.

36. Piovani D, Clavenna A, Cartabia M, Bonati M; Interregional Italian Drug Utilisation Group. Antibiotic and anti-asthmatic drug prescriptions in Italy: geographic patterns and socio-economic determinants at the district level. Eur J Clin Pharmacol. 2014;70(3):331-337.

37. de Jong J, Bos JH, de Vries TW, de Jong-van den Berg LT. Use of antibiotics in rural and urban regions in the Netherlands: an observational drug utilization study. BMC Public Health. 2014;14:677.

38. Rapporto OsMed [webpage on the Internet]. 2016. Available from: http:// www.aifa.gov.it/content/luso-dei-farmaci-italia-rapporto-osmed-2016. Accessed May, 2017. Italian [with English abstract].

39. ESAC-Net Surveillance Data. Summary of the Latest Data on Antibiotic Consumption in the European Union. 2017. Available from: https://ecdc. europa.eu/sites/portal/files/documents/Final_2017_EAAD_ESACNet_Summary-edited\%20-\%20FINALwith\%20erratum.pdf. Accessed February, 2017.

40. Sianesi B. The returns to education: macroeconomics. J Econ Surv. 2003;17(2):157-200. 
41. Gonzalez-Gonzalez C, Lopez-Vazquez P, Vazquez-Lago JM, et al; GREPHEPI Group. Effect of physicians' attitudes and knowledge on the quality of antibiotic prescription: a cohort study. PLoS One. 2015;10(10):e0141820.

42. Gaygisiz U, Lajunen T, Gaygisiz E. Socio-economic factors, cultural values, national personality and antibiotics use: a cross-cultural study among European countries. J Infect Public Health. 2017;10(6): 755-760.

43. Illario M, Vollenbroek-Hutten M, Molloy DW, Menditto E, Iaccarino G, Eklund P. Active and healthy ageing and independent living. J Aging Res. 2015;2015:542183.

44. Bousquet J, Bewick M, Cano A, et al. Building bridges for innovation in ageing: synergies between action groups of the EIP on AHA. J Nutr Health Aging. 2017;21(1):92-104.

45. Ministry of Health. Italian Medicines Agency. Circolare AIFA del 3 Agosto. 2007. Available from: http://xoomer.virgilio.it/pgiuff/osservazionali.pdf. Accessed May, 2017.

46. Iolascon $\mathrm{G}$, Gimigliano F, Moretti A, et al. Rates and reasons for lack of persistence with anti-osteoporotic drugs: analysis of the Campania region database. Clin Cases Miner Bone Metab. 2016;13(2): $127-130$.
47. Menditto E, Guerriero F, Orlando V, et al. Self-assessment of adherence to medication: a case study in Campania region community-dwelling population. J Aging Res. 2015;2015:682503.

48. Orlando V, Guerriero F, Putignano D, et al. Prescription patterns of antidiabetic treatment in the elderly. Results from Southern Italy. Curr Diabetes Rev. 2015;12(2):100-106.

49. Cammarota S, Falconio LM, Bruzzese D, et al. Lower rate of cardiovascular complications in patients on bolus insulin analogues: a retrospective population-based cohort study. PLoS One. 2013;8(11):e79762.

50. Scala D, Menditto E, Caruso G, et al. Are you more concerned about or relieved by medicines? An explorative randomized study of the impact of telephone counseling by pharmacists on patients' beliefs regarding medicines and blood pressure control. Patient Educ Couns. 2018;101(4):679-686.

51. Putignano D, Bruzzese D, Orlando V, Fiorentino D, Tettamanti A, Menditto E. Differences in drug use between men and women: an Italian cross sectional study. BMC Womens Health. 2017;17(1):73.

52. Guerriero F, Orlando V, Monetti VM, Russo V, Menditto E. Biological therapy utilization, switching, and cost among patients with psoriasis: retrospective analysis of administrative databases in Southern Italy. Clinicoecon Outcomes Res. 2017;9:741-748.

\section{ClinicoEconomics and Outcomes Research}

\section{Publish your work in this journal}

ClinicoEconomics and Outcomes Research is an international, peerreviewed open-access journal focusing on health technology assessment, pharmacoeconomics and outcomes research in the areas of diagnosis, medical devices, and clinical, surgical and pharmacological intervention. The economic impact of health policy and health systems
Submit your manuscript here: https://www.dovepress.com/clinicoeconomics-and-outcomes-research-journal

\section{Dovepress}

organization also constitute important areas of coverage. The manuscript management system is completely online and includes a very quick and fair peer-review system, which is all easy to use. Visit http://www.dovepress.com/testimonials.php to read real quotes from published authors. 